\title{
Dynamiques du développement de la pisciculture dans deux régions du Brésil : une approche comparée
}

\author{
Newton José Rodrigues da Silva ${ }^{1}$ \\ Jean-Eudes Beuret ${ }^{2}$ \\ Olivier Mikolasek ${ }^{3}$ \\ Guy Fontenelle ${ }^{4}$ \\ Lionel Dabbadie ${ }^{5}$ \\ Jérôme Lazard ${ }^{6}$ \\ Maria Iñez Espagnoli Geraldo Martins ${ }^{7}$ \\ 1 Coordenadoria de Assistência Técnica Integral \\ Escritório de Desenvolvimento Rural \\ de São Paulo \\ Avenida Bartolomeu de Gusmão, 192 \\ Ponta da Praia \\ 11030-600 - Santos, SP . \\ Brésil \\ <newtonrodrigues@uol.com.br> \\ 2 Département économie rurale \\ et gestion Agrocampus-Ouest \\ S 84215 \\ 65 , rue de St Brieuc \\ Rennes \\ France \\ <jean-eudes.beuret@agrocampus-ouest.fr> \\ ${ }^{3}$ Cirad \\ UR "Aquaculture et gestion \\ des ressources aquatiques" \\ TA B-20/01 \\ Avenue Agropolis \\ 34398 Montpellier Cedex 5 \\ France \\ <olivier.mikolasek@cirad.fr> \\ 4 Pôle halieutique UMR 0985 Inra \\ Agrocampus Ouest \\ CS 84215 \\ 65, rue de St Brieuc \\ 35042 Rennes Cedex \\ <guy.fontenelle@agrocampus-ouest.fr> \\ ${ }^{5}$ Cirad \\ UR "Aquaculture et gestion \\ des ressources aquatiques" \\ TA B-20/01 \\ Avenue Agropolis \\ 34398 Montpellier Cedex 5 \\ France \\ ${ }^{6}$ Cirad \\ TA B-20/01 \\ Avenue Agropolis \\ 34398 Montpellier \\ <jerome.lazard@cirad.fr> \\ 7 Universidade Estadual Paulista \\ Faculdade de Ciências Agrárias e Veterinárias \\ Via de Acesso Prof. Paulo Donato Castellane \\ s/n rural \\ 14884-900 - Jaboticabal, SP - \\ Brésil \\ <minezesp@fcav.unesp.br>
}

Tirés à part : O. Mikolasek

\begin{abstract}
Résumé
Dans une perspective historique, le développement de la pisciculture a été comparé dans deux régions du Brésil, en mobilisant le concept de Système local d'innovation (SLI) et les référentiels théoriques de la sociologie de la traduction et des économies de la grandeur. Les résultats de l'analyse montrent que dans la vallée du Ribeira, les relations au sein du réseau sociotechnique de la pisciculture ont seulement des caractéristiques marchandes, et il n'y a pas de coordination et d'alignement des actions entre les composantes du SLI : de ce fait, le réseau a été dans l'incapacité de créer des innovations techniques et organisationnelles face à la crise économique. Dans la haute vallée de l'Itajai, le réseau s'est construit sur la base de relations marchandes, civiques et de confiance. Les composantes des pôles de compétence du SLI, en interaction, présentent une coordination et un alignement, et le réseau de la pisciculture a été capable d'innovations techniques et organisationnelles pour faire face aux crises économiques et à caractère climatique.
\end{abstract}

Mots clés : Brésil ; pisciculture ; sociologie de la traduction ; système local d'innovation.

Thèmes : économie et développement rural ; pêche et aquaculture ; production animale.

\section{Abstract \\ The dynamics of fish farming development in two Brazilian valleys: A comparative approach}

Based on a historical approach, fish farming development was compared in two differentiated Brazilian areas. This study mobilizes the concept of Innovative Local System (ILS) together with the theoretical framework of the Sociology of Innovation and of the Economies of Size. We found that all the relationships within Ribeira Valley are mainly commercial based. There is neither coordination nor linking actions among the components of ILS. Hence, the "network" was unable to create technological or organizational innovations capable of facing any economic crisis. Conversely, in the Itajai High Valley, the socio-technological fish farming network has been developed on social, commercial, and trustful relationships. All interactions among the components of the ILS were coordinated to each other which allowed this network to react when economic or climatic threats threatened the sustainability of fish farming.

Key words: Brazil; fish culture; innovative local systems; sociology of innovation.

Subjects: animal production; economy and rural development; fishing and aquaculture.

$\mathrm{O}$ uels sont les facteurs qui permettent à une même activité de se développer ici, alors qu'elle régresse ailleurs, alors que les conditions naturelles et de marché ne peuvent expliquer à elles seules des trajectoires d'évolution différentes? Quel rôle jouent les poli- tiques publiques dans ces phénomènes? Nous avons tenté de répondre à ces questions et d'en tirer des enseignements pour les politiques publiques à partir d'une analyse menée dans deux régions, au Brésil. En effet, la pisciculture brésilienne s'est développée de manière différente dans la 
vallée de la Ribeira (VR), État de São Paulo, et la haute vallée de l'Itajaí (HVI), État de Santa Catarina. Dans la première région, la pisciculture n'a pas été capable d'innover pour surmonter la crise économique à laquelle elle était confrontée. Au contraire, dans la seconde région, la pisciculture se renforça grâce à des innovations techniques et organisationnelles, en réponse à plusieurs crises économiques et à caractère climatique. Forts de ce constat, nous nous sommes attachés à analyser et comparer les processus de développement, avec une perspective historique, dans les deux régions. L'objectif est d'identifier les facteurs responsables de l'échec ou de la réussite de la pisciculture et, de façon plus générale, d'identifier les facteurs qui déterminent la capacité des acteurs locaux d'une filière à innover pour apporter une réponse collective à des situations de crise et aux enjeux du développement de leurs activités.

Nous commencerons par présenter les deux régions ainsi que la démarche d'analyse et les principaux concepts sur lesquels elle repose, en particulier le concept de Système local d'innovation (SLI) selon Bureth et Llerena (1992) et les référentiels théoriques de la sociologie de la traduction (Callon, 1986) et des économies de la grandeur (Boltanski et Thévenot, 1991). Nous analyserons ensuite les dynamiques de développement de la pisciculture dans les deux régions étudiées. Pour finir, nous en déduirons des enseignements quant aux facteurs qui ont agi positivement et négativement sur le développement de la pisciculture, en nous intéressant en particulier au rôle joué par les politiques publiques.

\section{Concepts}

\section{et méthode d'analyse}

\section{Lieux d'étude et méthodologie}

Les deux régions étudiées ont été retenues après avoir constaté l'existence de trajectoires de développement très différentes de la pisciculture : dès lors, une analyse comparative semblait très opportune pour l'analyse des déterminants de telles trajectoires. Localisée dans le sudest de l'État de São Paulo, la VR (figure 1) est constituée de 23 municípios occupant $17382 \mathrm{~km}^{2}$, avec 4211312 habitants en
2000 (IBGE, 2003). Cette région montagneuse présente des écosystèmes très diversifiés constitués principalement de marais, d'un système estuarien-lagunaire et d'une forêt peu modifiée par les hommes sur des pentes raides. La principale banane. La pisciculture y est pratiquée par 664 exploitants (IEA, 1997). La population rurale est composée de descendants de japonais, de communautés noires et d'immigrants de diverses régions brésiliennes.

Dans la région centrale de l'État de Santa Catarina, la HVI (figure 1) regroupe 26 municipios occupant une surface de $6715,5 \mathrm{~km}^{2}$, avec 233770 habitants en 2000 (IBGE, 2003). Le relief est très varié : vallées profondes, plateaux limités par les escarpements de ces vallées et plaines inondables. Les principales activités agricoles sont la culture du tabac, de l'oignon, du maïs, du riz, du manioc et l'élevage de bovins, de porcs et de poulets. En 2001, la pisciculture commerciale était présente dans 396 exploitations (Souza Filho et al., 2002). La population est constituée principalement par des descendants d'immigrants allemands et italiens.

Afin de réaliser une analyse comparative, la même démarche d'analyse a été mise en œuvre dans les deux régions. En 2003, les trajectoires de la pisciculture ont été étudiées dans chacune des régions au moyen d'entretiens auprès de 13 personnes clés dans la VR et 19 dans la HVI, d'une analyse des documents officiels et activité économique est la culture de la

d'une enquête par questionnaires auprès de 20 pisciculteurs et ex-pisciculteurs de chaque région. Le choix des exploitants a été réalisé sur la base d'une typologie prenant en compte les caractéristiques techniques de la pisciculture et la destination de la production. L'approche historique a été choisie pour analyser les facteurs qui ont agi et interagi dans la construction de l'activité.

\section{Le SLI, un réseau sociotechnique}

Notre étude repose sur l'hypothèse que la compétitivité d'un réseau sociotechnique, c'est-à-dire sa capacité à innover et à réagir aux événements qui affectent les activités de ses membres, est directement liée aux interactions entre les pôles de compétence qui composent le SLI (figure 2). En effet, selon Callon (1986), l'innovation ne s'impose pas du fait de ses qualités propres, mais c'est le réseau qui la porte qui lui permet ou non de le faire. Dans le cas présent, à la suite de Bureth et Llerena (1992), nous considérons un SLI composé de quatre pôles : la production, la formation, la recherche et le financement. L'intensité et la qualité des interactions entre les composantes de chaque pôle de ce système déterminent sa capacité à porter l'innovation. Ces interactions peuvent être instables ou reposer sur des accords. Différents au départ, les intérêts des acteurs convergent, à travers un

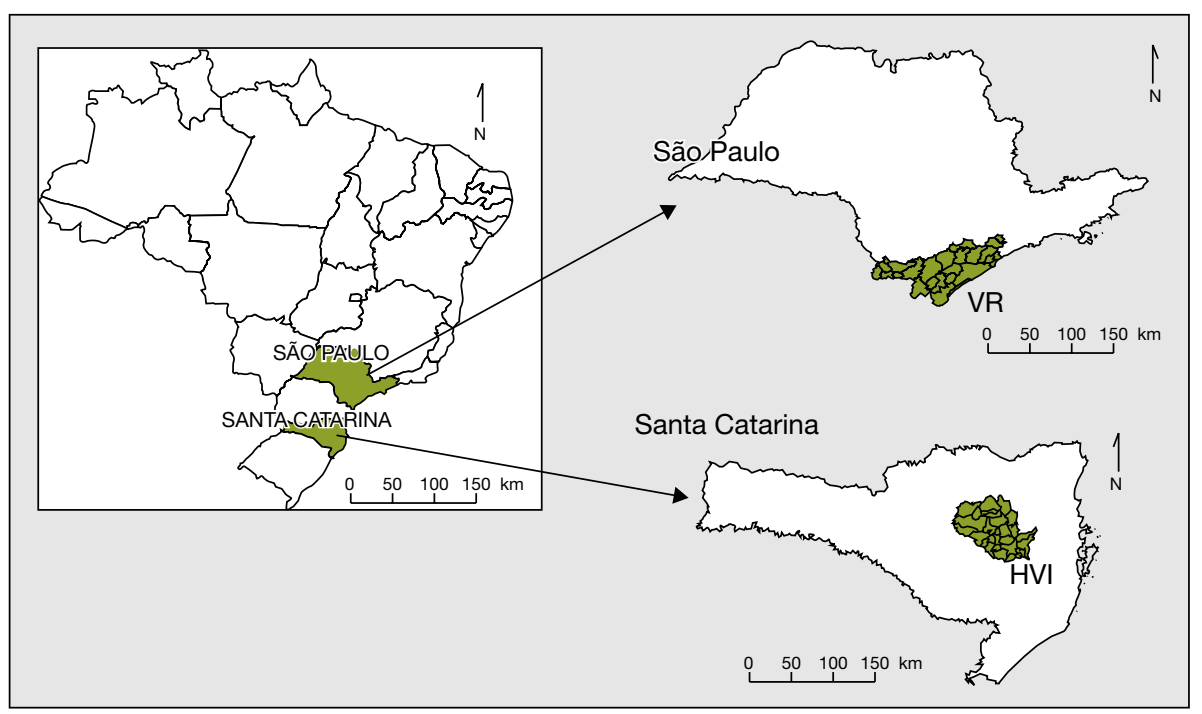

Figure 1. Localisation de la vallée du Ribeira et haute vallée de Itajaí dans les États de São Paulo et Santa Catarina du Brésil.

Figure 1. Localization of Ribeira Valley and the Itajaí High Valley in the States of São Paulo and Santa Catarina (Brazil). 


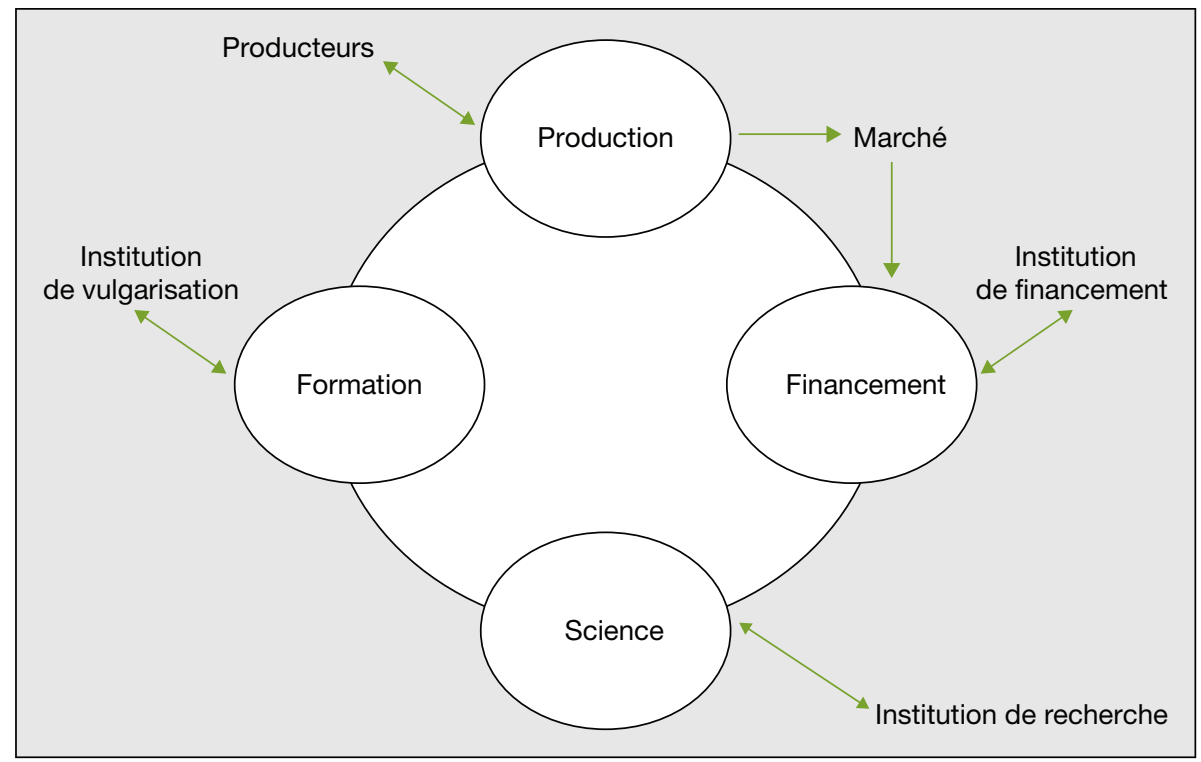

Figure 2. Représentation du Système local d'innovation et de ses interactions avec les quatre pôles de compétence.

Figure 2. Representation of the Local Innovative System and its interactions with the four poles of competence.

processus de traduction, vers un point de passage obligé (PPO) (Callon, 1986) et forment le noyau du réseau qui soutient l'innovation. Le PPO peut être un lieu, la formalisation de règles partagées ou une déclaration commune. Les acteurs qui, au départ, avaient des intérêts différents, et dont les relations étaient perturbées par des controverses, commencent à travailler ensemble pour la résolution d'un même problème. Finalement, les controverses favorisent le processus d'alignement des intérêts particuliers dans un projet d'ensemble minimum (Boltanski et Thévenot, 1991). Au cour de ces processus, la traduction est le fait d'un ou de plusieurs traducteurs : il s'agit d'un acteur social capable d'établir une compréhension mutuelle entre les acteurs concernés par le problème, ce qui favorise l'émergence de scénarios de coopération.

La coopération, lorsqu'elle parvient à se construire, s'inscrit dans des principes, valeurs de référence que Boltanski et Thévenot (1991) dénomment les grandeurs. Pour ces auteurs, la grandeur marchande est le monde des intérêts particuliers. Cette grandeur se caractérise par la concurrence, la captation de clientèle, l'obtention des meilleurs prix et de l'avantage maximum dans les transactions. La grandeur civique est caractérisée par l'intérêt collectif, qui dépasse les intérêts particuliers. La grandeur domestique est définie, d'une part, par l'attachement des personnes aux valeurs familiales et aux us et coutumes et, d'autre part, à l'établissement de relations de confiance. Elle se base sur la proximité entre les acteurs, que celle-ci existe d'emblée ou se construise au gré de leurs interactions.

\section{Construction}

\section{de la pisciculture dans la VR}

\section{1-1983 : émergence de l'activité, sans soutien externe}

La pisciculture a débuté en 1931 dans la VR, avec l'introduction de la carpe commune (Cyprinus carpio) par des immigrants japonais (da Silva et al., 2005). L'alimentation des poissons était réalisée avec des sous-produits agricoles, et la production était pour l'essentielle autoconsommée. Il a fallu attendre la fin des années 1970 pour que les producteurs sollicitent et bénéficient d'une assistance technique de la part du gouvernement de l'État. L'objectif du projet était de transformer cette pisciculture en une activité commerciale. Mais l'action publique n'a consisté qu'à implanter une unité de production d'alevins chez un unique exploitant plus aisé que les autres. Elle s'est soldée par un échec, car conduite hors du réseau sociotechnique en construction: l'ensemble des pisciculteurs n'a pas été associé à cette initiative qu'ils ne se sont pas appropriée.

\section{4-1991 : construction d'un SLI}

Cette période a été marquée par une controverse nationale autour du modèle technique de développement agricole brésilien. Très critiquée, la Révolution verte était perçue comme une modernisation conservatrice, provoquant l'exode rural et la concentration de la terre. Avec la victoire électorale des forces qui étaient à l'origine de la critique, un ensemble d'actions a été mis en œuvre pour favoriser la petite agriculture. À partir de 1984, la pisciculture a bénéficié d'une assistance technique et de distributions d'alevins de carpe commune et de tilapia (Oreochromis niloticus). Les vulgarisateurs spécialisés dans la pisciculture ont organisé des rencontres régionales, mettant en relation différents acteurs (pisciculteurs, techniciens et décideurs) et stimulant ainsi le débat sur les problèmes posés par cette activité. En 1989, le gouvernement de l'État a implanté un centre de recherche en aquaculture.

Ces actions ne se sont pas limitées à la diffusion de techniques. L'un des techniciens ayant débuté comme vulgarisateur, puis comme chercheur, a quitté le service public pour devenir pisciculteur. Ce parcours lui a permis de devenir le traducteur du processus, en devenant une passerelle entre la production, la formation et la recherche : à l'échelle d'un même individu, trois pôles de compétence du SLI étaient ainsi représentés. À Juquià, un município de la VR, un SLI complet a émergé en 1991. Celui-ci réunissait un groupe de pisciculteurs innovateurs ayant créé une association de producteurs, une ligne de crédit du municipio et l'action de chercheurs, actifs dans le domaine de la formation. Un fabricant d'aliments extrudés résidant à $300 \mathrm{~km}$ de Juquià intégra ensuite le réseau. Il existait alors une véritable coopération entre les acteurs sociaux.

\section{2-1997 :}

\section{expansion du SLI}

Cette période est marquée par l'expansion du SLI de Juquiá vers l'ensemble de la vallée. Le principal facteur déclenchant le processus a été une forte augmentation 
de la demande en poissons vivants à travers des parcours de pêche privés localisés dans l'agglomération de São Paulo. La pisciculture devient une activité très rémunératrice pour les producteurs. Le nombre d'espèces élevées augmente, mais sans système technique porté collectivement. Plus de cinq associations se créent. Néanmoins, elles s'engagent surtout dans l'achat des aliments et d'alevins hors de la vallée et jouent un rôle d'intermédiaires dans la commercialisation des poissons vivants. Les techniques d'élevage de poissons sont diffusées principalement par les techniciens des entreprises qui produisent l'aliment extrudé. Les services publics de vulgarisation n'interviennent pas dans le réseau. Les chercheurs assurent le transfert des propositions techniques au profit des entreprises d'aliments.

\section{8-2003 : déclin}

Le prix de l'aliment, principal composant du coût de production des poissons, augmente de manière importante. Simultanément, la faillite de nombreux parcours de pêche privés entraîne une baisse du prix d'achat des poissons aux pisciculteurs ainsi que des impayés. Le réseau de la pisciculture affronte sa première crise d'ordre économique. En raison de l'absence d'interactions entre les quatre pôles de compétence du SLI, il se montre incapable de créer les innovations techniques et organisationnelles qui lui auraient permis de surmonter la crise. En effet, les acteurs du réseau ne sont alors liés que par des relations marchandes : ces relations sont directement affectées par l'évolution des rapports de prix, et en l'absence de projets collectifs assortis d'un plan d'action et d'objectifs communs, les associations de producteurs deviennent inactives, et le réseau se déstructure.

\section{Construction}

\section{de la pisciculture}

\section{dans la HVI}

\section{Émergence de l'activité dans l'ouest de l'État, impulsée par les pouvoirs publics}

Dans la HVI, le système d'élevage de poissons pratiqué a été fortement influencé par les expériences localisées dans l'ouest du Santa Catarina. Cette région constitue un noyau de développement de la pisciculture pour l'ensemble de l'État. La sécheresse de 1977 constitue le facteur décisif. En 1980, le gouvernement lance un programme de construction de réservoirs d'irrigation. En dix ans, plus de 5000 réservoirs sont construits. Ce programme et l'assistance technique, tous les deux publics, coordonnent leurs actions pour promouvoir l'adoption de la pisciculture par l'élevage de la carpe commune, selon des références techniques chinoises. Les effluents de porcs sont utilisés pour fertiliser les eaux des réservoirs. Les techniciens des organismes gouvernementaux, en partenariat avec les agriculteurs, construisent le SLI de la pisciculture. Les vulgarisateurs et les chercheurs des organismes publics de l'État sont les porte-parole et les traducteurs du réseau sociotechnique émergeant.

\section{7-1993 : émergence de la pisciculture dans la HVI}

Cette période se caractérise par une meilleure coordination entre les pisciculteurs innovateurs du município de Trombudo central et la dynamique existant dans la région ouest de l'État. Celle-ci résulte de l'action d'un vulgarisateur de l'Empresa de Pesquisa Agropecuaria e Extensão Rural de Santa Catarina (EPAGRI), spécialisé en pisciculture. Les innovations de l'Ouest de l'État sont ainsi adoptées dans la HVI, la plus importante étant l'introduction des carpes chinoises (Hypophthalmichthys molitrix, Aristichthys nobilis et Ctenopharyngodon idella) pour pratiquer la polyculture. Cela conduit à une plus grande utilisation des déjections de porcs dans l'élevage de poissons. Le vulgarisateur local, avec les autres vulgarisateurs et chercheurs de l'Ouest et du Moyen Ouest, a servi de traducteur, en assurant le lien entre les pôles de recherche, de production et de formation du SLI, créant ainsi les conditions favorables à son émergence.

\section{4-1996 : construction d'un SLI à Agrolândia (HVI)}

En 1994, la première association municipale de pisciculteurs voit le jour à Trombudo central. Néanmoins, l'adoption de la pisciculture devient progressivement plus importante chez les éleveurs de porcs du município voisin, Agrolândia. L'arrivée du vulgarisateur, l'appui de la Chambre de commerce et de l'industrie locale dans le développement de l'activité et l'engagement de la préfecture engendrent une forte mobilisation sociale, dont les objectifs sont de créer un débouché commercial sûr, d'adopter une espèce de poisson mieux acceptée par le marché et, ainsi, de consolider la pisciculture comme activité commerciale.

En 1995, les pisciculteurs adoptent le tilapia monosexe mâle. Son élevage se fait en polyculture avec l'utilisation des déjections de porcs. L'entrée de cette espèce dans le réseau sociotechnique permet d'envisager un projet d'unité de transformation de poissons. Le vulgarisateur d'EPAGRI intéresse au projet l'entreprise Pamplona qui travaille en intégration avec les éleveurs de porcs. En 1996, l'implantation de l'unité de transformation de poissons par Pamplona facilite l'adoption de l'association porcs-poissons dans la HVI. La même année, grâce à l'action du vulgarisateur d'EPAGRI, un entrepreneur local développe un aliment granulé pour le tilapia. Après un test, cet aliment est adopté. Simultanément, l'aérateur est introduit. Le système d'élevage de poisson d'Agrolândia, foyer des innovations techniques, est adopté par d'autres producteurs dans toute la région. Sa diffusion est assurée, de fait, par des porte-parole que sont les vulgarisateurs de l'EPAGRI et les producteurs innovateurs. Le SLI de la pisciculture commerciale s'est développé à Agrolândia et est devenu un PPO pour les différents acteurs, c'est-à-dire une proposition dans laquelle tous se reconnaissent, à laquelle ils adhèrent et qui rend possible leur convergence autour d'un même SLI. La traduction est réalisée par les vulgarisateurs de l'EPAGRI.

\section{7 : controverse environnementale et évolution du système par traduction}

En novembre 1996, l'Associação de Preservação do Meio Ambiente do Alto Vale do Itajai (APREMAVI) se porte partie civile contre les diverses agences gouvernementales. D'après l'APREMAVI, la pisciculture intégrée à l'élevage de porc serait une source de pollution des rivières et la principale cause de la prolifération de la simulie borrachudo (Simulium pertinax). Cette ONG élabore et transmet un rapport (APREMAVI, 1996) auprès de nombreux destinataires, dont la Banque mondiale, leur demandant de prendre 
position. La Banque mondiale est le principal bailleur de fonds du Programme des microbassins hydrographiques dans l'État de Santa Catarina, programme qui contient une forte composante environnementale. Cette controverse provoque des craintes chez les pisciculteurs quant à l'avenir de leur activité et interrompt son développement. La direction de l'EPAGRI renforce l'encadrement technique de la pisciculture dans la région pour affronter ce débat dans les meilleures conditions.

À la suite de ces plaintes engageant le Programme des microbassins hydrographiques, dont l'EPAGRI a la maitrise d'œuvre, la Banque mondiale envoie une mission pour étudier la situation et émettre un avis technique. Une commission composée de l'EPAGRI et de l'APREMAVI analyse le document produit par cette mission (MISSÃO BIRD/FAO, 1997) et définit les actions pouvant rapprocher les intérêts des deux parties en discussion. À partir de novembre 1997, la pratique de la pisciculture intégrée à l'élevage de porcs est autorisée sous réserve de se conformer aux principales règles suivantes : le contrôle total des entrées et sorties d'eau des bassins de pisciculture et l'absence de renouvellement continu de l'eau ; la vidange lente du bassin lors des récoltes de poissons et le maintien du tiers final du niveau d'eau qui ne peut pas être relâché dans l'environnement ; un nombre de porcs par unité de surface inférieur à 60 individus par hectares ; seul l'EPAGRI est habilité à fournir un appui technique à l'implantation des projets autorisés; la formation des producteurs et des techniciens en pisciculture au sujet de la législation environnementale; l'édition de matériel didactique sur les normes de production de poissons et le suivi de la qualité de l'eau des microbassins.

La mission de la Banque mondiale a joué le rôle de traducteur, et les agents de l'EPAGRI celui de porte-parole de la pisciculture. Les règles qui définissent l'élevage intégré poissons-porcs ont évolué par traduction, permettant le glissement d'un système vers un autre, sans crise majeure.

\section{8-2003 : \\ expansion de la pratique de la pisciculture}

Les vulgarisateurs de l'EPAGRI, en relation avec les leaders de la pisciculture, préparent un plan d'action pour le développement de la pisciculture. Les projets proposés envisagent la formation des producteurs et des vulgarisateurs généralistes, un programme de recherche et la consolidation des organisations des producteurs. Pour accéder aux financements, chaque exploitation doit obtenir une autorisation sur la base d'un projet spécifique. Grâce à l'engagement des producteurs, des vulgarisateurs et du chercheur, la mise en ouvre du plan d'action contribue de manière décisive à la consolidation de la pisciculture.

En 2000, la rigueur exceptionnelle de l'hiver déclenche la mortalité de 761 t de poissons (Tamassia, 2000). Le chercheur de l'EPAGRI conclut que si la baisse de température avait déclenché le processus, elle n'était pas déterminante. En fait, la stratification thermique de la colonne d'eau durant le jour ne permettait pas aux poissons d'échapper aux conditions défavorables de certaines couches de cette même colonne. La réaction à cette crise est de recommander l'utilisation d'aérateurs durant la journée pour homogénéiser la colonne d'eau. Ces nouvelles connaissances acquises encouragent les pisciculteurs à poursuivre leur activité.

Mais un autre événement provoque un grand découragement chez les producteurs: l'unité de transformation de poissons cesse ses activités à la suite des accusations portées par APREMAVI. Néanmoins, des solutions sont peu à peu trouvées en commercialisant la production auprès d'usines de transformation du littoral de Santa Catarina, de transporteurs de poissons ou de parcours de pêche dépendant de la municipalité. Le réseau se montre capable d'innover pour surmonter les crises.

\section{Réseau sociotechnique} et facteurs

\section{de développement} piscicole

\section{dans les deux régions}

Le tableau 1 montre les caractéristiques des territoires et des réseaux sociotechniques de la pisciculture dans les deux régions étudiées.

\section{Proximité socioculturelle des acteurs : le poids de l'histoire}

Le processus d'occupation des territoires a fortement influencé le développement de la pisciculture. Dans la VR, il n'existe pas de proximité culturelle entre les pisciculteurs, à l'exception de ceux qui appartiennent à la colonie nippone. Les producteurs sont issus de différentes origines géographiques et professionnelles. Leur installation dans la région est le plus souvent récente. Cette situation rend plus difficile l'établissement de relations de confiance entre les pisciculteurs. Dans la HVI, l'occupation du territoire est principalement le fait des communautés allemande et italienne. La proximité culturelle entre les immigrés et leurs descendants est à la fois liée à leurs origines communes et à une histoire partagée au cours de la colonisation de leur nouveau pays. Elle a produit de la confiance entre les producteurs, favorisant notamment la vie associative. Aujourd'hui, ceux-ci appartiennent aux troisième et quatrième générations des premiers émigrants. Ces facteurs ont été essentiels pour établir et consolider des relations basées sur des grandeurs civiques et domestiques.

\section{Lorsque la controverse stimule l'innovation}

Dans la VR, la controverse a été de faible intensité, extérieure au territoire et de caractère politique sans lien direct avec l'activité piscicole. Dans la HVI, la controverse a été au contraire de grande intensité, interne au territoire et directement liée à la pérennité de l'activité piscicole. La construction de l'innovation semble avoir été stimulée par l'intensité de la controverse. Ainsi, dans la HVI, l'innovation s'est rapidement propagée en réponse à la controverse environnementale. En revanche, dans la VR, les acteurs ne se sont retrouvés autour d'un PPO que six ans après la controverse et sa résolution.

\section{Traducteur, un rôle crucial dans l'adoption de l'innovation}

Dans la VR, le município de Juquiá a été le centre du réseau sociotechnique qui s'est formé. Le traducteur, successivement vulgarisateur, chercheur et producteur, a couvert dans ses activités professionnelles les trois pôles du SLI, ce qui lui a 


\section{Tableau 1. Comparaison des dynamiques de développement de la pisciculture dans la vallée du Ribeira et la haute vallée de I'Itajaí.}

Table 1. Comparison of dynamics of fishfarming development in the Ribeira Valley and the Itajaí High Valley.

\begin{tabular}{|c|c|c|}
\hline Caractéristiques & Vallée du Ribeira & Haute vallée de I'Itajaí \\
\hline Occupation du territoire & $\begin{array}{l}\text { Population autochtone, colonisation japonaise, } \\
\text { investisseurs agricoles de diverses origines }\end{array}$ & $\begin{array}{l}\text { Colonisation allemande et italienne, agriculture } \\
\text { familiale }\end{array}$ \\
\hline \multirow[t]{2}{*}{ Type de controverse } & Faible : externe au territoire ; politique & Forte : interne au territoire ; environnementale \\
\hline & $\begin{array}{l}\text { Conséquence : projet diffusionniste } \\
\text { de la pisciculture (1984) }\end{array}$ & $\begin{array}{l}\text { Conséquence : établissement de règles pour } \\
\text { pratiquer l'activité (1997) }\end{array}$ \\
\hline \multirow[t]{2}{*}{ PPO } & Formation d’un SLI à Juquiá & Premier PPO : formation d'un SLI à Agrolândia \\
\hline & & $\begin{array}{l}\text { Second PPO : définition de pratiques } \\
\text { socialement acceptables }\end{array}$ \\
\hline \multirow[t]{2}{*}{ Traducteur } & Professionnel qui a agi sur les pôles du SLI & Traducteur du premier PPO : vulgarisateur \\
\hline & & $\begin{array}{l}\text { Traducteur du second PPO : mission de la } \\
\text { Banque mondiale }\end{array}$ \\
\hline $\begin{array}{l}\text { SLI : noyau du réseau } \\
\text { sociotechnique }\end{array}$ & Juquiá en 1990 & Agrolândia (1994-1998) \\
\hline Techniques utilisées & $\begin{array}{l}\text { Dépendent de ressources exogènes: } \\
\text { aliment extrudé }\end{array}$ & $\begin{array}{l}\text { Valorisation de ressources endogènes: } \\
\text { déjections de porcs }\end{array}$ \\
\hline Pôle de formation SLI & $\begin{array}{l}\text { Formation initiale, } \\
\text { puis techniciens des fabricants d'aliments }\end{array}$ & Apprentissage et vulgarisateurs \\
\hline Pôle de recherche SLI & $\begin{array}{l}\text { Unité de recherche implantée. } \\
\text { Action extérieure au réseau }\end{array}$ & $\begin{array}{l}\text { Recherche-développement chez les exploitants. } \\
\text { Action à l'intérieur du réseau }\end{array}$ \\
\hline Pôle de financement du SLI & Priorité des infrastructures & $\begin{array}{l}\text { Financement de la recherche et, surtout, de la } \\
\text { formation }\end{array}$ \\
\hline \multirow[t]{3}{*}{ Pôle de production du SLI } & $\begin{array}{l}\text { Faible organisation des producteurs. } \\
\text { Relation liée aux opportunités du marché }\end{array}$ & $\begin{array}{l}\text { Organisation de producteurs forte. Relation de } \\
\text { coopération, de confiance et marchande }\end{array}$ \\
\hline & $\begin{array}{l}\text { Absence de proximité géographique } \\
\text { entre les acteurs de la filière }\end{array}$ & $\begin{array}{l}\text { Proximité géographique } \\
\text { entre les acteurs de la filière }\end{array}$ \\
\hline & Absence de proximité culturelle entre les acteurs & Proximité culturelle entre les acteurs \\
\hline Marché & $\begin{array}{l}\text { Parcours de pêche } \\
\text { dans l'agglomération de São Paulo }\end{array}$ & $\begin{array}{l}\text { Parcours de pêche locaux et d'autres régions. } \\
\text { Transformation locale. Exportation }\end{array}$ \\
\hline \multirow[t]{6}{*}{ Réseau } & Marchand & Civique, domestique et marchand \\
\hline & Peu d'apprentissage & Grand apprentissage \\
\hline & Baisse de la vigilance & Grande vigilance \\
\hline & Baisse de la transparence & Grande transparence \\
\hline & Baisse de capacité de réaction & Grande capacité de réaction \\
\hline & Baisse de la durabilité & Grande durabilité \\
\hline Nature du processus & Diffusionniste & Constructiviste \\
\hline
\end{tabular}

SLI : Système local d'innovation ; PPO : point de passage obligatoire.

permis de jouer ce rôle de façon naturelle, car il maîtrisait les langages des uns et des autres. Dans la HVI, le município d'Agrolândia a été le centre du SLI et du réseau sociotechnique qui s'est formé autour de lui. Le vulgarisateur de l'EPAGRI a joué le rôle de traducteur, puis la mission de la Banque mondiale a assuré une traduction entre le monde de l'environnement et le SLI. La diffusion de l'innovation a été rendue possible par les garanties offertes aux producteurs pour investir dans l'association de l'élevage du porc et de la piscicul- ture : la qualité et la crédibilité de la traduction ont joué un rôle clé.

\section{Des techniques de production coconstruites : un atout pour leur adaptation}

Dans la VR, malgré une grande diversité d'espèces, l'élevage en monoculture prédomine. Le granulé extrudé est le principal intrant utilisé, ce qui crée une dépen- dance vis-à-vis d'une ressource exogène au territoire. Il ne s'agit pas d'un système d'élevage construit collectivement, mais de la diffusion d'un modèle technique. Dans la HVI, la polyculture de tilapias avec les carpes chinoises et l'utilisation d'aérateurs sont généralisés. Les intrants sont principalement constitués par les effluents porcins. Utilisé uniquement dans la période finale d'élevage, le granulé pressé est fabriqué par une entreprise locale. Le système d'élevage de poissons a été construit par les acteurs du 
réseau, en valorisant les conditions physiques, économiques et sociales locales. Cette coconstruction s'est faite sur la base du SLI qui, lui-même, va conduire l'adaptation de ce système d'élevage face aux événements extérieurs.

\section{Continuité de l'action publique et intégration sociale}

Le pôle "formation", dans la VR, a été porté par les vulgarisateurs, puis des chercheurs et enfin des techniciens liés à des usines d'aliments. Ce pôle a été actif dans le début du processus de développement de la pisciculture et pendant son apogée, avec la formation initiale de producteurs et le transfert de technologies. Cependant, dans la dernière période, ce développement a été conduit hors du réseau sociotechnique. L'agence gouvernementale responsable de la vulgarisation agricole de l'État de São Paulo (CATI) n'a pas détaché de vulgarisateurs spécialisés pour appuyer la pisciculture mais seulement des vulgarisateurs généralistes. Ainsi, la formation des producteurs n'a pas été constante et n'a pas interagi avec les autres pôles du SLI. Dans la HVI, le pôle "formation" a été investi principalement par l'EPAGRI, agence du gouvernement de l'État de Santa Catarina. L'action publique en matière de formation a été présente dans tout le développement de l'activité. Il s'agit d'un processus d'apprentissage qui a impliqué des acteurs des différents pôles du SLI. Pendant la controverse environnementale, le renforcement du pôle " formation " par des vulgarisateurs spécialisés supplémentaires a augmenté les interactions avec les pôles "production" et "recherche ". La continuité a joué un rôle essentiel.

Pour ce qui est du pôle " recherche ", dans la VR, les vulgarisateurs ont d'abord collaboré avec des instituts de recherche externes au territoire. Puis, le gouvernement de l'État a implanté une unité de recherche (CEPAR). Au début, les chercheurs privilégient le transfert de technologies en répondant aux attentes individuelles des pisciculteurs les plus riches. Par la suite, les thèmes de recherche ne s'inscrivent plus dans les préoccupations des producteurs, et le pôle "recherche" n'agit pas pour faciliter la construction des innovations techniques et organisationnelles. Dans la HVI, les vulgarisateurs de Trombudo central bénéficient, au départ, de l'apport des chercheurs qui interviennent dans l'Ouest de l'État. Puis, le transfert d'un chercheur vers la HVI a consolidé le réseau. Ces nouvelles compétences ont été mobilisées pour faire face à la controverse environnementale, et les recherches conduites au sein du réseau ont permis de résoudre les problèmes causés par l'hiver et la fermeture de l'unité de transformation de poissons.

Les composantes du pôle de production du SLI, dans la VR, sont situées hors du territoire, notamment les principaux producteurs d'alevins et les usines d'aliments extrudés. Les entreprises n'ont pas établi de relations directes avec les producteurs de poissons, et les coordinations sont guidées seulement par le marché. Par ailleurs, les interactions entre les producteurs sont limitées en raison du manque de proximité géographique. Ainsi, l'éloignement existant entre les différentes composantes de la production explique, en partie, l'incapacité du réseau à réagir face à la crise économique. Dans la HVI, les différentes composantes de la production sont situées dans le territoire ou dans les lieux proches. Les producteurs d'alevins, le fabricant d'aliment, les transporteurs de poissons, les unités de traitement de poissons et les fabricants d'équipements sont en contact permanent avec les pisciculteurs, les vulgarisateurs et le chercheur. Les proximités géographique et culturelle, ainsi que l'engagement des différents acteurs dans le développement de l'activité, favorisent l'apprentissage et les interactions entre les pôles de SLI. La localisation des différentes composantes du pôle " production " dans le territoire est fondamentale pour le développement d'un apprentissage collectif. Les liens entre les différentes composantes augmentent la coordination et l'alignement du réseau, améliorant sa compétitivité.

\section{Un facteur essentiel : l'intégration locale du pôle financement du SLI}

Dans la VR, bien que l'État ait investi dans les infrastructures piscicoles, la majorité des actions a été réalisée hors de la dynamique locale existante. Le cas le plus emblématique est celui de la pisciculture de la vallée de l'Etá : le gouvernement de l'État a concentré les ressources financières sur un promoteur extérieur au territoire pour implanter un élevage de crevettes d'eau douce au détriment des producteurs locaux qui élevaient des poissons. En revanche, dans la HVI, les ressources financières sont utilisées pour la formation des producteurs et des vulgarisateurs généralistes, ainsi que pour l'affectation de techniciens spécialisés et d'un chercheur en pisciculture. Les recherches conduites chez les pisciculteurs se sont montrées très efficaces. Les interactions entre producteurs, vulgarisateurs et chercheurs, grâce à un investissement financier intégré à la dynamique locale, ont favorisé les apprentissages et l'innovation.

\section{Conclusion}

L'analyse comparative que nous avons menée permet de faire ressortir les principaux déterminants des réactions différenciées des deux réseaux sociotechniques, face aux crises et à la nécessité d'innover. La nature des relations entre les acteurs qui composent le réseau sociotechnique de la pisciculture de la VR est strictement d'ordre marchand, et le réseau n'a pas la capacité de réagir face à la crise économique. Dans la HVI, les relations entre les membres du réseau sont fondées sur des grandeurs marchandes mais aussi domestiques et civiques. La circulation des informations est facilitée par la proximité géographique, sociale, culturelle et professionnelle qui existe entre les acteurs de la pisciculture. Des coopérations s'établissent entre les producteurs et les autres pôles du SLI, incluant vulgarisateurs et chercheurs: c'est ce qui permet au réseau de réagir et de surmonter les crises à caractère économique ou climatique.

Quels enseignements en déduire quant aux politiques publiques à mettre en œuvre? L'acteur public devra s'intéresser aux quatre pôles du SLI et veiller à leur intégration locale. Il devra par ailleurs soutenir les traductions lorsqu'elles existent ou les faire émerger. La traduction est souvent assurée par la vulgarisation agricole, qui peut jouer un rôle important pour faire ressortir un ensemble d'intérêts communs, favoriser la convergence des acteurs autour de ces intérêts et favoriser l'émergence de relations qui ne soient pas seulement marchandes. L'investissement dans la formation est au cour du processus, et les actions de recherche et le financement doivent être insérés dans les dynamiques locales existantes. Il s'agit d'intégrer tous les acteurs, producteurs, chercheurs, formateurs et financeurs, dans une même dynamique et dans un apprentissage collectif. 


\section{Références}

APREMAVI. O sol não pode ser tapado com a peneira. Rio do Sul : APREMAVI, 1996.

Boltanski L, Thevenot L. De la justification. Les économies de la grandeur. Paris: Gallimard, 1991.

Bureth A, Llerena P. Système local d'innovation : approche théorique et premiers résultats empiriques. In: Actes du colloque industrie et territoire : les systèmes productifs localisés, 21 et 22 octobre 1992. Grenoble: Institut de recherche économique sur la production et le développement, 1992.
Callon M. Éléments pour une sociologie de la traduction: la domestication des coquilles Saint-Jacques et des marins-pêcheurs dans la baie de Saint-Brieuc. Annee Sociol 1986; 36: 169-208.

da Silva NJR, Beuret JE, Mikolasek O, Fontenelle G, Dabbadie L, Martins M. Dynamiques de développement de la pisciculture et politiques publiques dans la vallée du Ribeira, Etat de São Paulo (Brésil). Cah Agric 2005; 14 : 59-63.

IBGE. Capturado no site www.ibge.com em 11/09/2003.

IEA. Censo Agropecuário no Estado de São Paulo. Informações Econômicas IEA 1997; 27 : 7-140.
MISSÃO BIRD/FAO. Complemento à ajuda memória: aspectos ambientais do sistema de produção peixe-suíno na região do Alto Vale do Itajaí. Missão de acompanhamento ao Projeto Microbacias, realizada entre 22 a 29 de setembro de 1997.

Souza Filho J, Schappo CL, Tamassia STJ. Estudo de competitividade da piscicultura no Alto Vale do Itajaí. Florianópolis: Instituto CEPA/SC ; Epagri, 2002.

Tamassia STJ. Inverno 2000: avaliação e providências. InfoPeixe Vale do Itajaí. Rio do Sul: Associação Regional de Aqüicultores do Alto Vale do Itajaí, 2000. 